\title{
Parallelizations on products of spheres and octonionic geometry
}

https://doi.org/10.1515/coma-2019-0007

Received January 15, 2019; accepted March 9, 2019

Abstract: A classical theorem of Kervaire states that products of spheres are parallelizable if and only if at least one of the factors has odd dimension. Two explicit parallelizations on $S^{m} \times S^{2 h-1}$ seem to be quite natural, and have been previously studied by the first named author in [32]. The present paper is devoted to the three choices $G=G_{2}$, $\operatorname{Spin}(7)$, $\operatorname{Spin}(9)$ of $G$-structures on $S^{m} \times S^{2 h-1}$, respectively with $m+2 h-1=7,8,16$ and related with octonionic geometry.

Keywords: $G_{2}$, Spin(7), Spin(9), octonions, parallelizations on spheres, locally conformally parallel.

MSC: Primary 53C26, 53C27, 53C35, 57R25.

\section{Introduction}

Let $\left(M^{d}, g\right)$ be an oriented Riemannian manifold, $\nabla$ its Levi-Civita connection, and $G$ a closed subgroup of $\mathrm{SO}(d)$. Whenever $G$ is the stabilizer of some tensor $\eta$ on the Euclidean space $\mathbb{R}^{d}$, a $G$-structure on $M$ gives rise to a global tensor $\eta$ on $M$, and the covariant derivative $\nabla \eta$ can be viewed as a section of the vector bundle

$$
\mathcal{W}=T^{\star} M \otimes \mathfrak{g}^{\perp}
$$

referring to the orthogonal decomposition $\mathfrak{s o}(d)=\mathfrak{g} \oplus \mathfrak{g}^{\perp}$, [40, Corollary 2.2]. The covariant derivative $\nabla \eta$ is called the intrinsic torsion of the $G$-structure, and can be used to classify $G$-structures in the following way.

The action of $G$ splits $\mathcal{W}$ into irreducible components $\mathcal{W}=\mathcal{W}_{1} \oplus \cdots \oplus \mathcal{W}_{k}$. According to this decomposition, $G$-structures on $M$ can be classified into (at most) $2^{k}$ classes, each class corresponding to those $G$-structures whose intrinsic torsion lifts to a section of one of the subspaces $\mathcal{W}_{i_{1}} \oplus \cdots \oplus \mathcal{W}_{i_{l}}$ of $\mathcal{W}$ :

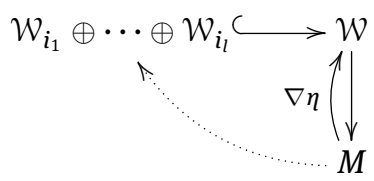

In this framework, the holonomy condition turns out to be the most restrictive one, since the condition of Riemannian holonomy contained in $G$ is equivalent to $\eta$ being parallel with respect to $g$, that is, the intrinsic torsion is zero. On the opposite side, we have $G$-structures of general type, that is, when the intrinsic torsion does not lift to any proper $G$-invariant subspace of $\mathcal{W}$.

Whenever $G \neq \mathrm{SO}(d)$, the standard $G$-representation $\mathbb{R}^{d}$ always appears as an irreducible component in the decomposition of $\mathcal{W}$. A $G$-structure lifting to this component is said to be locally conformally parallel, because in this case $g$ is locally conformal to Riemannian metrics with holonomy contained in $G$.

^Corresponding Author: Maurizio Parton: Dipartimento di Economia, Università di Chieti-Pescara, Viale della Pineta 4, I-65129 Pescara, Italy, E-mail: parton@unich.it

Paolo Piccinni: Dipartimento di Matematica, Sapienza-Università di Roma, Piazzale Aldo Moro 2, I-00185 Roma, Italy, E-mail: piccinni@mat.uniroma1.it 
The prototype example of this decomposition is the A. Gray and L. Hervella decomposition [23], with $\eta$ the Kähler 2-form and $G=\mathrm{U}(n)$, dealing with almost Hermitian structures on a $M^{2 n}$. When $n \geq 3$, the space $\mathcal{W}$ splits into four $\mathrm{U}(n)$-irreducible components, giving rise to sixteen classes of almost Hermitian manifolds. The number of classes reduces to four when $n=2$.

Further examples have been studied in 1980s. Notably, M. Fernandez and A. Gray [17] considered $G=$ $\mathrm{G}_{2} \subset \mathrm{SO}(7)$ with $\eta$ the associative exterior 3-form. The outcome is four irreducible components $\mathcal{W}=\mathcal{W}_{1} \oplus$ $\mathcal{W}_{2} \oplus \mathcal{W}_{3} \oplus \mathcal{W}_{4}$, giving rise to (a-priori) sixteen classes. Also, M. Fernandez [14] studied $G=\operatorname{Spin}(7)$ with $\eta$ the Cayley 4-form, obtaining two irreducible components $\mathcal{W}=\mathcal{W}_{1} \oplus \mathcal{W}_{2}$. A structure theorem for locally conformally parallel $\mathrm{G}_{2}$ and Spin(7)-structures has been proved by S. Ivanov and the present authors, [25].

The irreducible components description for $\mathrm{G}_{2}$ and $\operatorname{Spin}(7)$ in terms of symmetries of $\nabla \eta$ has been simplified by F. Cabrera, [7, 8], using the fact that $\nabla \eta$ is completely determined by $d \eta$ and $d^{*} \eta$ for $\mathrm{G}_{2}$ and by $d \eta$ for Spin(7), [40]. Moreover, Cabrera gives explicit examples of locally conformally parallel structures on product of spheres: a $\operatorname{Spin}(7)$-structure $\phi_{S^{7} \times S^{1}}$ on $S^{7} \times S^{1}$, [7], and a $\mathrm{G}_{2}$-structure $\varphi_{S^{6} \times S^{1}}$ on $S^{6} \times S^{1}$, [9].

The choice of Spin(9) $\subset$ SO(16) Riemannian holonomy is known since the 1960s to be possible only on the (symmetric) Cayley plane $\mathrm{F}_{4} / \mathrm{Spin}(9)$ and its non-compact dual, [2]. A systematic study of non-integrable Spin(9)-structures on Riemannian $\left(M^{16}, g\right)$ was initiated by Th. Friedrich in 1999, [20-22]. Here the classification is pursued through a description of $\mathfrak{s p i n}(9)^{\perp}$ as $\Lambda^{3}\left(E^{9}\right)$, for a suitably defined vector bundle $E^{9}$ locally spanned by 9 auto-adjoint, anti-commuting involutions. In this paper, Friedrich gives an example of a Spin(9)-structure $\Phi_{S^{15} \times S^{1}}$ on $S^{15} \times S^{1}$.

Friedrich's description of non-integrable Spin(9)-structures appears to be very useful when dealing with structures related to Spin(9), and was used in several papers by the present authors, [29, 33-39], some also with L. Ornea and V. Vuletescu.

A beautiful and careful description of intrinsic torsion, together with its history and its relation to nonintegrable geometries, can be found in [1].

A very special $G$-structure appears whenever $M$ is parallelizable. In fact, a parallelization on $M^{d}$ is a vector bundle isomorphism between $T M$ and the trivial bundle $M \times \mathbb{R}^{d}$ or, equivalently, a section of the principal bundle $\mathrm{SO}(M)$. As such, it defines a $\{1\}$-structure on $M$, and therefore a $G$-structure for any closed $G \in \operatorname{SO}(d)$. We say in this case that the $G$-structure is associated to the parallelization. If $G$ is the stabilizer of a tensor $\eta$ on $\mathbb{R}^{d}$, a global tensor $\eta$ on $M$ is given by the above isomorphism $M \times \mathbb{R}^{d} \simeq T M$. Despite its trivial definition, differential properties of $\eta$ can be non-trivial, because they depend on the structure equations of the parallelization. Note that this is the modern approach to invariant structures on nilmanifolds and solvmanifolds, see the vast literature on this topic, e.g. [4, 11, 14-16, 18, 19].

A product of spheres is parallelizable if and only if one of the factors has odd dimension. This was proved by M. Kervaire [27], see also the simpler proof given by E. B. Staples [41]. Under this hypothesis, a product of spheres can then be equipped with any $G$-structure compatible with its dimension, and the properties of the $G$-structure depend on the choice of the parallelization.

The present paper develops a topic that was in the doctoral thesis of the first named author [30]. Namely, we take into account two parallelizations on $S^{m} \times S^{n}$, when $n$ is odd, denoted by $\mathcal{B}$ and $\mathcal{P}$. A thorough description of $\mathcal{B}$ and $\mathcal{P}$, together with the underlying geometric idea and their structure equations, can be found in $[6,31,32]$. Note that we consider $\mathcal{B}$ only for $n=1,3$, while in the original paper of M. Bruni it was defined also when $n=5,7$.

In the mentioned thesis, and in [31, 32], the almost Hermitian and almost hyper-Hermitian structures associated to $\mathcal{B}$ and $\mathcal{P}$, when the sum $m+n$ permits, had been studied. In particular, the almost Hermitian structures turn out to be the Calabi-Eckmann structures, and thus belong to $\mathcal{W}_{3} \oplus \mathcal{W}_{4}-\left(\mathcal{W}_{3} \cup \mathcal{W}_{4}\right)$, [10, Theorem 4.4].

In this paper we study the further choices of $G$-structures with $G=G_{2}$, Spin(7) and $\operatorname{Spin}(9)$, in the framework of the intrinsic torsion. Accordingly, we assume the dimension of $S^{m} \times S^{n}$ to be 7,8 and 16, respectively. This is a summary of the results obtained.

- $n=1$ : the $G_{2}$, Spin(7) and $\operatorname{Spin}(9)$-structures associated to $\mathcal{B}$ belong to the locally conformally parallel class, and they coincide with Cabrera's $G_{2}$ and Spin(7)-structures $\varphi_{S^{6} \times S^{1}}, \phi_{S^{7} \times S^{1}}$, and with Friedrich's $\operatorname{Spin}(9)$-structure $\Phi_{S^{15} \times S^{1}}$. 
- $n=1$ : the $\mathrm{G}_{2}$ and $\operatorname{Spin}(7)$-structures associated to $\mathcal{P}$ belong to $\mathcal{W}$, and are thus of general type.

- $n>1$ : the $\mathrm{G}_{2}$ and Spin(7)-structures associated to $\mathcal{B}$ and $\mathcal{P}$ are of general type.

Note that the fact that the structures associated to $\mathcal{P}$ are of general type is not in contrast with the mentioned result about Calabi-Eckmann structures, because $\mathcal{W}_{3} \oplus \mathcal{W}_{4}-\left(\mathcal{W}_{3} \cup \mathcal{W}_{4}\right)$ represents the general type structures between the integrable complex structures.

Finally, we study the intrinsic torsion under the action of the orthogonal group $\mathrm{O}(m+n)$ and the symmetric group $\mathfrak{S}_{m+n}$.

- $n=1$ : the $\mathrm{G}_{2}$, $\operatorname{Spin}(7)$ and $\operatorname{Spin}(9)$-structures associated to parallelizations in the orthogonal orbit $\mathrm{O}(m+$ 1) $\mathcal{B}$ are isomorphic to $\varphi_{S^{6} \times S^{1}}, \phi_{S^{7} \times S^{1}}$ and $\Phi_{S^{15} \times S^{1}}$, respectively.

- $n=1$ : the $\mathrm{G}_{2}$-structures associated to parallelizations in the orthogonal orbit $\mathrm{O}(m+1) \mathcal{P}$ are of general type.

- $n=3$ : the $\mathrm{G}_{2}$ and Spin(7)-structures associated to parallelizations in the symmetric orbit $\mathfrak{S}_{m+3} \mathcal{B}$ are of general type.

- $n \geq 1$ : the $\mathrm{G}_{2}$ and $\operatorname{Spin}(7)$-structures associated to parallelizations in the symmetric orbit $\mathfrak{S}_{m+n} \mathcal{P}$ are of general type.

\section{Preliminaries}

\subsection{Two parallelizations on $S^{m} \times S^{n}$}

In this section, we briefly describe two parallelizations $\mathcal{B}$ and $\mathcal{P}$ on product of spheres $S^{m} \times S^{n}$, with odd $n$. Note that $\mathcal{B}$, proposed by M. Bruni in [6], is defined using a parallelization of $S^{n}$, that is, only for $n=1,3$ or 7 , and with a different argument for $n=5$. We will consider $\mathcal{B}$ only in the cases $n=1,3$. Instead, $\mathcal{P}$ is defined for every odd $n$, [30].

\section{The parallelization $\mathcal{B}$ on $\mathbf{S}^{\mathbf{m}} \times \mathbf{S}^{1}$ and $\mathbf{S}^{\mathrm{m}} \times \mathbf{S}^{3}$}

When $n=1$, the parallelization $\mathcal{B}$ at the point $\left(\left(x_{1}, \ldots x_{m+1}\right), \theta\right) \in S^{m} \times S^{1} \subset \mathbb{R}^{m+1} \times S^{1}$ is defined by referring to the following meridian vector fields:

$$
M_{i} \stackrel{\text { def }}{=} \text { orthogonal projection of } \partial_{x_{i}} \text { on } S^{m} \quad i=1, \ldots, m+1
$$

and to the unit tangent vector field $\partial_{\theta}$ of $S^{1}$. Then it is easy to see that

$$
\mathcal{B} \stackrel{\text { def }}{=}\left\{b_{1}, b_{2}, \ldots, b_{m+1}\right\} \stackrel{\text { def }}{=}\left\{\left(M_{1}, x_{1} \partial_{\theta}\right),\left(M_{2}, x_{2} \partial_{\theta}\right), \ldots,\left(M_{m+1}, x_{m+1} \partial_{\theta}\right)\right\}
$$

is a parallelization of $T_{\left(x_{1}, \ldots, x_{m+1}\right)} S^{m} \times T_{\theta} S^{1}$ which is orthonormal with respect to the standard product metric of $S^{m} \times S^{1}$.

Remark 2.1. The vector fields $\left\{|x| \partial_{x_{i}}\right\}_{i=1, \ldots, m+1}$ on $\mathbb{R}^{m+1} \backslash 0$ are projectable with respect to the universal covering map $\mathbb{R}^{m+1} \backslash 0 \rightarrow S^{m} \times S^{1}$ given by $p(x)=(x /|x|, \log |x| \bmod 2 \pi)$. It is easily proved that this is an alternative construction for $\mathcal{B}$ on $S^{m} \times S^{1}$.

The above construction of $\mathcal{B}$ on $S^{m} \times S^{1}$ relies on the never-vanishing vector field $T=\partial_{\theta}$ on $S^{1}$. In the case $S^{m} \times S^{3} \subset \mathbb{R}^{m+1} \times \mathbb{R}^{4}$, we choose one of the three never-vanishing vector fields on $S^{3}$ to mimic the same construction. Namely, say $e_{1}, e_{2}, e_{3}$ is an orthonormal frame in $T_{\left(y_{1}, \ldots, y_{4}\right)} S^{3}$, and choose $T=e_{1}$. Then:

$$
\mathcal{B} \stackrel{\text { def }}{=}\left\{b_{1}, b_{2}, \ldots, b_{m+1}, b_{m+2}, b_{m+3}\right\}=\left\{\left(M_{1}, x_{1} T\right),\left(M_{2}, x_{2} T\right), \ldots,\left(M_{m+1}, x_{m+1} T\right),\left(0, e_{2}\right),\left(0, e_{3}\right)\right\}
$$

is an orthonormal parallelization of $T_{\left(x_{1}, \ldots, x_{m+1}\right)} S^{m} \times T_{\left(y_{1}, \ldots, y_{4}\right)} S^{3}$. 


\section{The parallelization $\mathcal{P}$ on $\mathbf{S}^{\mathrm{m}} \times \mathbf{S}^{\mathrm{n}}$, $\mathbf{n}$ odd}

Let now $n$ be odd, say $n=2 h-1$, and $S^{m} \times S^{n} \subset \mathbb{R}^{m+1} \times \mathbb{R}^{2 h}$. Denote by $x_{i}$ and $y_{j}$ the coordinates of $\mathbb{R}^{m+1}$ and $\mathbb{R}^{2 h}$, respectively. Denote by $T$ the never-vanishing vector field on $S^{n}=S^{2 h-1}$ given by complex multiplication:

$$
T=\sum_{j=1}^{n+1} t_{j} \partial_{y_{j}}=-y_{2} \partial_{y_{1}}+y_{1} \partial_{y_{2}}-\cdots-y_{2 h} \partial_{y_{2 h-1}}+y_{2 h-1} \partial_{y_{2 h}} .
$$

Finally, denote by $M_{i}$ and $N_{j}$ the meridian vector fields on $S^{m}$ and $S^{n}$, respectively:

$$
\begin{aligned}
& M_{i} \stackrel{\text { def }}{=} \text { orthogonal projection of } \partial_{x_{i}} \text { on } S^{m} \quad i=1, \ldots, m+1, \\
& N_{j} \stackrel{\text { def }}{=} \text { orthogonal projection of } \partial_{y_{j}} \text { on } S^{n} \quad j=1, \ldots, n+1 \text {. }
\end{aligned}
$$

The parallelization $\mathcal{P}$ on $S^{m} \times S^{n}$ is then given by the vector fields:

$$
\begin{aligned}
p_{i} & \stackrel{\text { def }}{=} M_{i}+x_{i} T \quad i=1, \ldots, m-1, \\
p_{m-1+j} & \stackrel{\text { def }}{=} y_{j} M_{m}+t_{j} M_{m+1}+\left(t_{j} x_{m+1}+y_{j} x_{m}-t_{j}\right) T+N_{j} \quad j=1, \ldots, n+1 .
\end{aligned}
$$

The geometric idea for $\mathcal{P}$ is explained in [32], and is based on Staples' proof in [41]. Briefly, a rank one vector bundle $\langle T\rangle$ is detached from $T S^{n}=E \oplus\langle T\rangle$, and it is used to trivialize $T S^{m}$. Then, a rank two trivial summand from $T S^{m} \oplus\langle T\rangle$ is used to trivialize $E$. In the above construction of $\mathcal{P}$, the rank two trivial summand is spanned by the last two meridian vector fields of $S^{m}$, that is, $M_{m}$ and $M_{m+1}$. Of course, there is nothing special about $M_{m}$ and $M_{m+1}$, and one could instead choose any other pair of meridian vector fields.

\section{$\mathbf{S}^{\mathrm{m}} \times \mathbf{S}^{1}$ and $\mathbf{S}^{\mathrm{m}} \times \mathbf{S}^{3}$, change of basis and structure equations}

The following table gives the relation between $\mathcal{B}$ and $\mathcal{P}$ on $S^{m} \times S^{n}$, when $n=1$, 3:

\begin{tabular}{|c|c|c|c|}
\hline & $n=1$ & $n=3$ & $n>3$ \\
\hline $\mathcal{B}$ & $d b^{i}=\tau \wedge b^{i}, i=1, \ldots, m+1$ & $\begin{aligned} d b^{i}=b^{i} & \wedge \tau+2 x_{i} b^{m+2} \wedge b^{m+3}, i=1, \ldots, m+1 \\
d b^{m+2} & =2 b^{m+3} \wedge \tau \\
d b^{m+3} & =-2 b^{m+2} \wedge \tau\end{aligned}$ & \\
\hline $\mathcal{P}$ & $\begin{array}{c}d p^{i}=p^{i} \wedge \tau, i=1, \ldots, m-1 \\
d p^{m}=p^{m} \wedge \tau+p^{m+1} \wedge \tau \\
\quad d p^{m+1}=p^{m+1} \wedge \tau-p^{m} \wedge \tau\end{array}$ & (6) in Appendix & (6) in Appendix \\
\hline
\end{tabular}

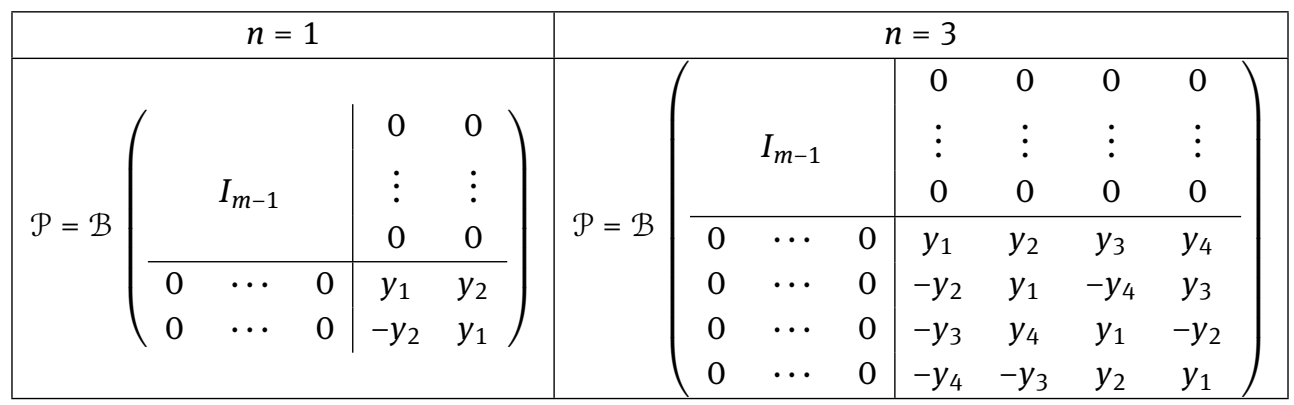

Denoting by $\left\{b^{i}\right\}$ and $\left\{p^{i}\right\}$ the dual bases of $\mathcal{B}=\left\{b_{i}\right\}$ and $\mathcal{P}=\left\{p_{i}\right\}$, the structure equations can be computed by a direct calculation, described in details in [30]. The final result is given in the following formulas: 
where $\tau$ is given by:

$$
\begin{aligned}
& \tau=\sum_{i=1}^{m+1} x_{i} b^{i} \quad \text { for } \mathcal{B} \\
& \tau=\sum_{i=1}^{m-1} x_{i} p^{i}+\left(x_{m} y_{1}-x_{m+1} y_{2}\right) p^{m}+\left(x_{m} y_{2}+x_{m+1} y_{1}\right) p^{m+1} \quad \text { for } \mathcal{P}
\end{aligned}
$$

Remark 2.2. The parallelization $\mathcal{P}$ for $n \geq 3$ is very twisted, and it is therefore given as a long formula in Appendix.

Remark 2.3. Both $\mathcal{B}$ and $\mathcal{P}$ (the first defined here only for $n=1,3$ ) are orthonormal parallelizations with respect to the product metric on $S^{m} \times S^{n}$. Acting by the symmetric group $\mathfrak{S}_{m+n}$ and by the orthogonal group $\mathrm{O}(m+n)$ preserves orthonormality, thus all the $G$-structures we look at in this paper are compatible with the product metric.

\subsection{Structures related to octonions.}

We recall here some basic facts on the octonions. Let $\left\{e_{1}, \ldots, e_{7}\right\}$ be the standard basis of $\mathbb{R}^{7}$, and $\left\{e^{1}, \ldots, e^{7}\right\}$ be the dual basis. One has then in $\mathbb{R}^{8}=\mathbb{R} \oplus \mathbb{R}^{7}$ the standard basis $\left\{1, e_{1}, \ldots, e_{7}\right\}$ and the dual basis $\left\{\lambda, e^{1}, \ldots, e^{7}\right\}$.

The non-associative normed algebra $\mathbb{O}$ of Cayley numbers is defined in $\mathbb{R}^{8}$ through the standard scalar product $\langle\cdot, \cdot\rangle$ and the multiplication rules $e_{i}^{2}=-1, e_{i} e_{j}=-e_{j} e_{i}$ and $e_{i} e_{i+1}=e_{i+3}$ for all cyclic permutation of $\{i, i+1, i+3\}$, where indices run in $\mathbb{Z}_{7}$.

With this choice, the standard quaternion subalgebra $\mathbb{H}$ is generated by $1, e_{1}, e_{2}, e_{4}$. We are here following the approach for example of $[3,5]$, and everything can be rephrased by ordering the octonion units as $\{1, i, j, k, e, i e, j e, k e\}=\left\{1, e_{1}, e_{2}, e_{4}, e_{3}, e_{7}, e_{5},-e_{6}\right\}$ (cf. for example [24, 26]).

The 3 -form $\varphi$ on $\mathbb{R}^{7}=\mathfrak{I} m(\mathbb{O})$ is defined as

$$
\varphi \stackrel{\text { def }}{=}\langle x, y z\rangle=\sum_{i \in \mathbb{Z}_{7}} e^{i, i+1, i+3},
$$

where from now on we use notations like $e^{i, i+1, i+3} \stackrel{\text { def }}{=} e^{i} \wedge e^{i+1} \wedge e^{i+3}$, and where $\mathrm{G}_{2}$ is the stabilizer of $\varphi$ in GL(8). Thus, for any ordered orthonormal basis $\mathcal{C}$ on an Euclidean vector space $V^{7}$, a $\mathrm{G}_{2}$-structure $\varphi_{\mathcal{C}}$ on $V$ is defined.

If * is the Hodge star on $\left(\mathbb{R}^{8},\langle\cdot, \cdot\rangle\right)$, it follows

$$
\star(\lambda \wedge \varphi)=-\sum_{i \in \mathbb{Z}_{7}} e^{i+2, i+4, i+5, i+6}=-\sum_{i \in \mathbb{Z}_{7}} e^{i, i+2, i+3, i+4},
$$

and the 4-form $\phi \stackrel{\text { def }}{=} \lambda \wedge \varphi+\star(\lambda \wedge \varphi)$ on $\mathbb{R}^{8}$ satisfies

$$
\phi=\lambda \wedge \sum_{i \in \mathbb{Z}_{7}} e^{i, i+1, i+3}-\sum_{i \in \mathbb{Z}_{7}} e^{i, i+2, i+3, i+4} .
$$

The Lie group $\operatorname{Spin}(7) \subset \mathrm{SO}(8)$ is the stabilizer of $\phi$, and again if $\mathcal{C}$ is any ordered orthonormal basis on an Euclidean vector space $V^{8}$, the above equation defines a Spin(7)-structure $\phi_{\mathcal{C}}$ on $V$.

\section{$3 G_{2}$ and Spin(7)-structures on products of spheres}

$A_{G_{2}}$-structure on a manifold $M^{7}$ is a reduction to $\mathrm{G}_{2} \subset \mathrm{GL}(7)$ of the structure group, and from $\mathrm{G}_{2} \subset \mathrm{SO}(7)$ one gets a Riemannian metric $g$. Since $\mathrm{G}_{2}$ is the stabilizer of $\varphi, \mathrm{a} \mathrm{G}_{2}$-structure gives rise to a global 3-form on $M^{7}$, 
at each point defined as (3) and called the associative 3-form. Conversely, such a 3-form on $M^{7}$ is equivalent to a $\mathrm{G}_{2}$-structure. Accordingly, a $\mathrm{G}_{2}$-structure is often identified with its 3-form.

Similarly, a Spin(7)-structure on a manifold $M^{8}$, namely, a reduction of the structure group GL(8) to $\operatorname{Spin}(7) \subset \operatorname{SO}(8)$, induces a Riemannian metric and, since $\operatorname{Spin}(7)$ is the stabilizer of $\phi$, a $\operatorname{Spin}(7)$-structure on $M^{8}$ can be identified with a global 4-form that can be locally written as $\phi$, called the Cayley 4-form.

Definition 3.1. Let $M$ be a seven or eight-dimensional manifold with a $\mathrm{G}_{2}$ or $\operatorname{Spin}(7)$-structure, respectively. Let $\varphi$ or $\phi$ be its associated differential form and let $\nabla$ be the Levi-Civita connection of the induced metric. The structure is said to be parallel if $\nabla \varphi=0$ or $\nabla \phi=0$, and locally conformally parallel if $\varphi$ or $\phi$ is locally conformal to local structures $\varphi_{\alpha}, \phi_{\alpha}$, which are parallel with respect to the local Levi-Civita connections they define.

$\mathrm{A} \mathrm{G}_{2}$-structure is parallel if and only if $d \varphi=d^{\star} \varphi=0$, and a Spin(7)-structure is parallel if and only if $d \phi=0$, [40]. Thus, one gets the following characterizations of locally conformally parallel $\mathrm{G}_{2}$ and $\operatorname{Spin}(7)$-structures, $[7,8]$.

Theorem 3.2. $A \mathrm{G}_{2}$-structure $\varphi$ on $M^{7}$ is locally conformally parallel if and only if there exists a closed $\tau \in$ $\Omega^{1}(M)$ such that $d \varphi=3 \tau \wedge \varphi, d^{\star} \varphi=4 \tau \wedge{ }^{\star} \varphi$. A Spin(7)-structure $\phi$ on $M^{8}$ is locally conformally parallel if and only if there exists a closed $\tau \in \Omega^{1}(M)$ such that $d \phi=\tau \wedge \phi$.

Proof. Let $\varphi$ be a locally conformally parallel $\mathrm{G}_{2}$-structure. Then for each $x \in M$, there exist a neighborhood $U$ of $x$ and a map $\sigma: U \rightarrow \mathbb{R}$ such that the local $\mathrm{G}_{2}$-structure $\varphi_{U}=e^{-3 \sigma} \varphi_{\left.\right|_{U}}$ is parallel with respect to its local Levi-Civita connection. One then obtains $d \varphi_{U}=d{ }^{\star}{ }_{U} \varphi_{U}=0$, where ${ }^{\star} U$ is the local Hodge star-operator associated to $\varphi_{U}$, and using these relations together with $e^{4 \sigma_{\star}}{ }_{U}=e^{3 \sigma_{\star}}$, one obtains $d \varphi_{\left.\right|_{U}}=3 d \sigma \wedge \varphi_{\left.\right|_{U}}, d^{\star} \varphi_{\left.\right|_{U}}=$ $4 d \sigma \wedge^{\star} \varphi_{\left.\right|_{U}}$. The closed 1-form $\tau$ locally defined by $d \sigma$ is easily seen to be global. The reverse implication is obtained the same way, once observed that since $\tau$ is closed then there exist local maps $\sigma: U \rightarrow \mathbb{R}$ such that $\tau_{\left.\right|_{U}}=d \sigma$. A similar argument applies for $\operatorname{Spin}(7)$.

Let $\varphi_{\mathcal{B}}, \phi_{\mathcal{B}}$ be the $\mathrm{G}_{2}$, $\operatorname{Spin}(7)$-structure on $S^{6} \times S^{1}, S^{7} \times S^{1}$ associated to $\mathcal{B}$, respectively, that is,

$$
\varphi_{\mathcal{B}} \stackrel{\text { def }}{=} \sum_{i \in \mathbb{Z}_{7}} b^{i, i+1, i+3}, \quad \phi_{\mathcal{B}} \stackrel{\text { def }}{=} b^{8} \wedge \sum_{i \in \mathbb{Z}_{7}} b^{i, i+1, i+3}-\sum_{i \in \mathbb{Z}_{7}} b^{i, i+2, i+3, i+4} .
$$

The following theorem describe $\varphi_{\mathcal{B}}$ and $\phi_{\mathcal{B}}$ in terms of the universal covering $p: \mathbb{R}^{m+1} \backslash 0 \rightarrow S^{m} \times S^{1}$, see Remark 2.1.

Theorem 3.3. The $\mathrm{G}_{2}$ and $\operatorname{Spin}(7)$-structures $\varphi_{\mathcal{B}}$ and $\phi_{\mathcal{B}}$ are locally conformally parallel. The local parallel structures are induced by $\varphi$, $\phi$ via $p: \mathbb{R}^{m+1} \backslash 0 \rightarrow S^{m} \times S^{1}$, for $m=6,7$ respectively.

Proof. The 3-form $\varphi=\sum_{i \in \mathbb{Z}_{7}} d x_{i} \wedge d x_{i+1} \wedge d x_{i+3}$ is parallel, and on $\mathbb{R}^{7} \backslash 0$ it is globally conformal to the $p$-invariant 3-form

$$
\varphi^{\prime}=\frac{1}{|x|^{3}} \sum_{i \in \mathbb{Z}_{7}} d x_{i} \wedge d x_{i+1} \wedge d x_{i+3}
$$

Observe that $\mathbb{R}^{7} \backslash 0$ is locally diffeomorphic to $S^{6} \times S^{1}$, and that $\varphi^{\prime}$ induces $\varphi_{\mathcal{B}}$, to end the proof in the $\mathrm{G}_{2}$ case. The $\operatorname{Spin}(7)$ case is similar.

Since $\mathcal{B}$ is orthonormal, the metric induced on $S^{6} \times S^{1}, S^{7} \times S^{1}$ by means of $\varphi_{\mathcal{B}}$, $\phi_{\mathcal{B}}$ is the product metric. Observe also that, since a parallel $\mathrm{G}_{2}$ or $\operatorname{Spin}(7)$-structure on a compact $M$ gives a non-trivial element in 3 or 4-dimensional cohomology, $S^{6} \times S^{1}$ and $S^{7} \times S^{1}$ cannot admit a parallel $\mathrm{G}_{2}$ and $\operatorname{Spin}(7)$-structure.

The same construction applies to seven and eight-dimensional products of spheres when $\mathcal{P}$ is chosen. On $S^{4} \times S^{3}, S^{5} \times S^{3}$ also $\mathcal{B}$ is available.

In [17], [14] the irreducible components of $\mathcal{W}$ are identified. It is useful to recall the following simplified defining relations, cf. [7], [8]. 
Lemma 3.4. The following types of $\mathrm{G}_{2}$-structures on $M^{7}$ can be characterized as follows:

- $\mathcal{W}_{1} \oplus \mathcal{W}_{2} \oplus \mathcal{W}_{3}$ if and only if $(\star d \varphi) \wedge \varphi=0$;

- $\mathcal{W}_{1} \oplus \mathcal{W}_{2} \oplus \mathcal{W}_{4}$ if and only if there exist $\alpha \in \Omega^{1}(M), f \in C^{\infty}(M)$ such that $d \varphi=\alpha \wedge \varphi+f^{\star} \varphi$;

- $\mathcal{W}_{1} \oplus \mathcal{W}_{3} \oplus \mathcal{W}_{4}$ if and only if there exists $\beta \in \Omega^{1}(M)$ such that $d^{\star} \varphi=\beta \wedge{ }^{\star} \varphi$;

- $\mathcal{W}_{2} \oplus \mathcal{W}_{3} \oplus \mathcal{W}_{4}$ if and only if $d \varphi \wedge \varphi=0$.

The following types of $\operatorname{Spin}(7)$-structures on $M^{8}$ are characterized as:

- $\mathcal{W}_{1}$ if and only if $\left({ }^{\star} d \phi\right) \wedge \phi=0$;

- $\mathcal{W}_{2}$ if and only if there exists $\alpha \in \Omega^{1}(M)$ such that $d \phi=\alpha \wedge \phi$.

Accordingly, $a G_{2}$ or Spin(7)-structure is said to be of general type if none of the above relations is satisfied.

Theorem 3.5. The $\mathrm{G}_{2}$-structure $\varphi_{\mathcal{P}}$ associated to the frame $\mathcal{P}$ on $S^{6} \times S^{1}$ is of general type.

Proof. The 3-form $\varphi_{\mathcal{P}}$ and the 4-form ${ }^{\star} \varphi_{\mathcal{P}}$ are given by

$$
\varphi_{\mathcal{P}}=\sum_{i \in \mathbb{Z}_{7}} p^{i, i+1, i+3}, \quad{ }^{\star} \varphi_{\mathcal{P}}=-\sum_{i \in \mathbb{Z}_{7}} p^{i, i+2, i+3, i+4} .
$$

Using formulas (2.1) for $\mathcal{P}$ and $n=1$ one obtains

$$
\begin{aligned}
d \varphi_{\mathcal{P}} & =3 \varphi_{\mathcal{P}} \wedge \tau-\left(p^{6,1,3}+p^{4,5,6}-p^{3,4,7}-p^{5,7,1}\right) \wedge \tau, \\
d^{\star} \varphi_{\mathcal{P}} & =-4^{\star} \varphi_{\mathcal{P}} \wedge \tau-\left(p^{7,1,3}+p^{4,5,7}+p^{3,4,6}+p^{5,6,1}\right) \wedge p^{2} \wedge \tau .
\end{aligned}
$$

A computation shows that none of the relations in Lemma 3.4 is satisfied, and $\varphi_{\mathcal{P}}$ is of general type.

The same conclusion holds for seven and eight-dimensional product of spheres equipped with the frame $\mathcal{P}$, and for $S^{4} \times S^{3}, S^{5} \times S^{3}$ with the frame $\mathcal{B}$ : in this case the computation is based on formulas (6) in Appendix, and has been checked with a computer calculation.

Theorem 3.6. The $\mathrm{G}_{2}$-structures associated to the frames $\mathcal{B}$ and $\mathcal{P}$ on $S^{4} \times S^{3}$ and to the frame $\mathcal{P}$ on $S^{2} \times S^{5}$ are of general type. The Spin(7)-structures associated to the frame $\mathcal{P}$ on $S^{7} \times S^{1}$, to the frames $\mathcal{B}$ and $\mathcal{P}$ on $S^{5} \times S^{3}$ and to the frame $\mathcal{P}$ on $S^{3} \times S^{5}$ are of general type.

The parallelization $\mathcal{B}$ for $n=1$ gives locally conformally parallel structures, that coincide with Cabrera's structures.

Theorem 3.7. The locally conformally parallel structures $\phi_{S^{7} \times S^{1}}$ and $\varphi_{S^{6} \times S^{1}}$ defined in [7] and [9] coincide with $\phi_{\mathcal{B}}$ and $\varphi_{\mathcal{B}}$, respectively.

Proof. Since $p: \mathbb{R}^{8} \backslash 0 \rightarrow S^{7} \times S^{1}$ is a local diffeomorphism and $p^{\star}\left(\phi_{\mathcal{B}}\right)=|x|^{-4} \phi$, we are left to prove that $p^{\star}\left(\phi_{S^{7} \times S^{1}}\right)=|x|^{-4} \phi$. Consider the versor field $N \stackrel{\text { def }}{=}|x|^{-1}\left(x_{1} \partial_{x_{1}}+\cdots+x_{8} \partial_{x_{8}}\right)$ on $\mathbb{R}^{8} \backslash 0$ and its dual 1-form $n \in \Omega^{1}\left(\mathbb{R}^{8} \backslash 0\right)$. Then, from the definition of $\phi_{S^{7} \times S^{1}}$ in [7]:

$$
p^{\star}\left(\phi_{S^{7} \times S^{1}}\right)=|x|^{-4}\left(n \wedge i_{N} \phi+{ }^{\star}\left(n \wedge i_{N} \phi\right)\right)
$$

and since the action of $\operatorname{Spin}(7)$ on $S^{7}$ is transitive, one obtains $n \wedge i_{N} \phi+{ }^{\star}\left(n \wedge i_{N} \phi\right)=\phi$. This completes the proof of the statement about $\operatorname{Spin}(7)$. To complete the proof, choose the embedding $S^{6} \times S^{1} \subset S^{7} \times S^{1}$ given by $x_{8}=0$. The normal vector field is then $\partial_{x_{8}}=b_{8}$, and from the definition of $\varphi_{S^{6} \times S^{1}}$ in [9], one obtains $\varphi_{S^{6} \times S^{1}} \stackrel{\text { def }}{=} i_{\partial_{x_{8}}} \phi_{S^{7} \times S^{1}}=i_{b_{8}} \phi_{\mathcal{B}}=\varphi_{\mathcal{B}}$. 


\section{The Spin(9)-structure on $S^{15} \times S^{1}$}

A Spin(9)-structure on a sixteen-dimensional Riemannian manifold $(M, g)$ is a reduction of the structure group of $M$ to Spin(9) $\subset \mathrm{SO}(16)$. According to [20], an equivalent definition is the datum of a rank 9 vector subbundle of End(TM), locally generated by self-dual anti-commuting involutions related on the intersections of their defining open sets by matrices of $\mathrm{SO}(9)$. This approach has been exploited in a series of papers of the present authors and coauthors [29, 33-39].

In particular, $\operatorname{Spin}(9)$ is the stabilizer of a canonical 8-form $\Phi \in \Lambda^{8}\left(\mathbb{R}^{16}\right)$, that can be constructed in several equivalent ways, cf. for example $[12,13,28,35]$. In particular, on a parallelizable $M^{16}$, any parallelization gives rise to such a global 8-form. One has therefore $\operatorname{Spin}(9)$ 8-forms on $S^{15} \times S^{1}, S^{13} \times S^{3}, S^{11} \times S^{5}, S^{9} \times S^{7}$, $S^{7} \times S^{9}, S^{5} \times S^{11}, S^{3} \times S^{13}, S^{1} \times S^{15}$ associated to $\mathcal{B}, \mathcal{P}$ and denoted by $\Phi_{\mathcal{B}}, \Phi_{\mathcal{P}}$, respectively.

Theorem 4.1. The Spin(9)-structure on $S^{15} \times S^{1}$ given by $\Phi_{\mathcal{B}}$ is locally conformally parallel. The local parallel Spin(9)-structures are induced by $\Phi$ via $p: \mathbb{R}^{16} \backslash 0 \rightarrow S^{15} \times S^{1}$.

Proof. This follows by the fact that $|x|^{-8} \Phi$ is $p$-invariant, globally conformal to $\Phi$, and induces $\Phi_{\mathcal{B}}$, as in the proof of Theorem 3.3.

We now briefly describe the approach of [20] to classes of $\operatorname{Spin}(9)$-structures. Let $\mathcal{R}$ be a $\operatorname{Spin}(9)$-structure on a 16-dimensional Riemannian manifold $M^{16}$, and denote by $\mathcal{F}(M)$ the principal orthonormal frame bundle. Then $\mathcal{R}$ is a subbundle of $\mathcal{F}(M)$ :

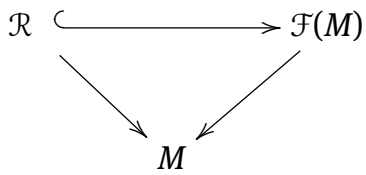

The Levi-Civita connection $Z: T(\mathcal{F}(M)) \rightarrow \mathfrak{s o}(16)=\mathfrak{s p i n}(9) \oplus \mathfrak{s p i n}(9)^{\perp}$ restricted to $T(\mathcal{R})$ decomposes into $Z^{\star} \oplus \Gamma$, where $Z^{\star}$ is a connection in the principal Spin(9)-fibre bundle $\mathcal{R}$, and $\Gamma \in \Omega^{1}\left(\mathcal{R} \times \operatorname{Spin}(9) \mathfrak{s p i n}(9)^{\perp}\right)=$ $\Omega^{1}\left(\Lambda^{3}(V)\right)$, where $V=V^{9} \stackrel{\text { def }}{=} \mathcal{R} \times \operatorname{Spin(9)} \mathbb{R}^{9}$. The irreducible components of $\Lambda^{1}(M) \otimes \Lambda^{3}(V)$ are described in [20]. In particular, one component is the 16-dimensional representation $\Lambda^{1}(M)$, that defines the nearly parallel $\operatorname{Spin}(9)$-structures. The action of $\operatorname{Spin}(9)$ on $S^{15}$ is transitive, with isotropy subgroup Spin(7), and this allows to define the principal Spin(7)-fibre bundle $\mathcal{R}_{S^{15} \times S^{1}} \operatorname{Spin}(9) \times S^{1} \rightarrow S^{15} \times S^{1}$, that in [20] is shown to be actually a nearly parallel Spin(7) $\subset$ Spin(9)-structure $\Phi_{S^{15} \times S^{1}}$ on $S^{15} \times S^{1}$.

Theorem 4.2. The nearly parallel Spin(9)-structure $\Phi_{S^{15} \times S^{1}}$ coincides with the locally conformally parallel $\operatorname{Spin}(9)$-structure $\Phi_{\mathcal{B}}$ associated to $\mathcal{B}$.

Proof. Consider the following diagram of $\operatorname{Spin}(7) \subset \operatorname{Spin}(9)$-structures:

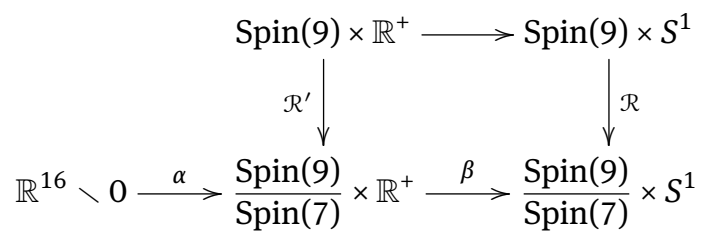

where $\alpha(x)=(x /|x|,|x|)$ and $\beta([g], \rho)=([g], \log \rho \bmod 2 \pi)$. Then $\beta \circ \alpha=p: \mathbb{R}^{16} \backslash 0 \rightarrow S^{15} \times S^{1}$ and the map $\alpha^{-1} \circ \mathcal{R}^{\prime}: \operatorname{Spin}(9) \times \mathbb{R}^{+} \rightarrow \mathbb{R}^{16} \backslash 0$ is a $\operatorname{Spin}(7) \subset \operatorname{Spin}(9)$-structure on $\mathbb{R}^{16} \backslash 0$. The pull-back $(\beta \circ \alpha)^{\star} \Phi_{\mathcal{B}} \in$ $\Omega^{8}\left(\mathbb{R}^{16} \backslash 0\right)$ gives by definition the admissible frame $\left\{|x| \partial_{x_{1}}, \ldots,|x| \partial_{x_{16}}\right\}$. A direct computation shows that this frame is admissible also for $\alpha^{-1} \circ \mathcal{R}^{\prime}$. 


\section{Orthogonal and symmetric orbits}

The representations of $\mathfrak{S}_{m+n}$ and $\mathrm{O}(m+n)$ on $\mathbb{R}^{m+n}$ give symmetric and orthogonal orbits of $G$-structures on $S^{m} \times S^{n}$. In this section we describe the following orbits:

- $\mathfrak{S}_{7} \varphi_{\mathcal{P}}$ on $S^{6} \times S^{1}, S^{4} \times S^{3}, S^{2} \times S^{5} ; \mathfrak{S}_{8} \phi_{\mathcal{P}}$ on $S^{7} \times S^{1}, S^{5} \times S^{3}, S^{3} \times S^{5}, S^{1} \times S^{7}$;

- $\mathfrak{S}_{7} \varphi_{\mathcal{B}}$ on $S^{4} \times S^{3} ; \mathfrak{S}_{8} \phi_{\mathcal{B}}$ on $S^{5} \times S^{3}$;

- $\mathrm{O}(7) \varphi_{\mathcal{B}}$ on $S^{6} \times S^{1} ; \mathrm{O}(8) \phi_{\mathcal{B}}$ on $S^{7} \times S^{1} ; \mathrm{O}(16) \Phi_{\mathcal{B}}$ on $S^{15} \times S^{1}$;

- $\mathrm{O}(7) \varphi_{\mathcal{P}}$ on $S^{6} \times S^{1}$.

Theorem 5.1. The symmetric orbits $\mathfrak{S}_{7} \varphi_{\mathcal{P}}$ of $\mathrm{G}_{2}$-structures on $S^{6} \times S^{1}, S^{4} \times S^{3}, S^{2} \times S^{5}, \mathfrak{S}_{8} \phi_{\mathcal{P}}$ of $\operatorname{Spin}(7)$ structures on $S^{7} \times S^{1}, S^{5} \times S^{3}, S^{3} \times S^{5}, S^{1} \times S^{7}, \mathfrak{S}_{7} \varphi_{\mathcal{B}}$ of $\mathrm{G}_{2}$-structures on $S^{4} \times S^{3}, \mathfrak{S}_{8} \phi_{\mathcal{B}}$ of $\operatorname{Spin}(7)$-structures on $S^{5} \times S^{3}$ are of general type.

Proof. Use theorem 3.4 together with the structure equations (2.1).

Lemma 5.2. Let $A \in \mathrm{O}(m+1)$. Then $A: \mathbb{R}^{m+1} \backslash 0 \rightarrow \mathbb{R}^{m+1} \backslash 0$ is $p$-invariant, and the induced diffeomorphism $f_{A}: S^{m} \times S^{1} \rightarrow S^{m} \times S^{1}$ is given by $(x, \theta) \mapsto(A(x), \theta)$. Moreover, the matrix of $d f_{A}$ with respect to the basis $\mathcal{B}$ on $S^{m} \times S^{1}$ is $A$.

Proof. Linear algebra computation. The universal covering map $p$ is given in Remark 2.1.

Theorem 5.3. The $\mathrm{G}_{2}$, $\operatorname{Spin}(7)$, $\operatorname{Spin}(9)$-structures on $S^{6} \times S^{1}, S^{7} \times S^{1}, S^{15} \times S^{1}$ in the orthogonal orbits $\mathrm{O}(7) \varphi_{\mathcal{B}}$, $\mathrm{O}(8) \phi_{\mathcal{B}}, \mathrm{O}(16) \Phi_{\mathcal{B}}$ are isomorphic to $\varphi_{\mathcal{B}}, \phi_{\mathcal{B}}, \Phi_{\mathcal{B}}$, respectively.

Proof. Let $\varphi_{A(\mathcal{B})} \in \mathrm{O}(7) \varphi_{\mathcal{B}}$. Then Lemma 5.2 gives $f_{A}^{\star}\left(\varphi_{A(\mathcal{B})}\right)=\varphi_{\mathcal{B}}$. Same argument for Spin(7) and Spin(9).

Note that Lemma 5.2 does not hold for $\mathcal{P}$ on $S^{m} \times S^{1}$, because of the twisting of $p_{m}, p_{m+1}$. One gets:

Theorem 5.4. The $\mathrm{G}_{2}$-structures on $S^{6} \times S^{1}$ in the orthogonal orbit $\mathrm{O}(7) \varphi_{\mathcal{P}}$ are of general type.

Proof. Let $A=\left(a_{i, j}\right) \in \mathrm{SO}(7)$, and let $\left\{q^{1}, \ldots, q^{7}\right\}$ be the parallelization on $S^{6} \times S^{1}$ induced by $A$ :

$$
q^{i} \stackrel{\text { def }}{=} \sum_{j=1}^{7} a_{i, j} p^{j} \quad i=1, \ldots, 7 .
$$

Let $\tau=-y_{2} d y_{1}+y_{1} d y_{2}$ a 1-form on $S^{6} \times S^{1}$, and let $u_{i}$ be its coordinates with respect to $\left\{q^{1}, \ldots, q^{7}\right\}$ :

$$
\tau=u_{1} q^{1}+\cdots+u_{7} q^{7} .
$$

Then

$$
\varphi_{A(\mathcal{P})}=\sum_{i \in \mathbb{Z}_{7}} q^{i, i+1, i+3}, \quad{ }^{\star} \varphi_{A(\mathcal{P})}=-\sum_{i \in \mathbb{Z}_{7}} q^{i, i+2, i+3, i+4},
$$

and using the structure equations (2.1) one obtains

$$
\begin{aligned}
d \varphi_{A(\mathcal{P})} & =3 \varphi_{A(\mathcal{P})} \wedge \tau+\sum_{i \in \mathbb{Z}_{7}}\left(\left(a_{i, 6} p^{7}-a_{i, 7} p^{6}\right) q^{i+1, i+3}-\left(a_{i+1,6} p^{7}-a_{i+1,7} p^{6}\right) q^{i, i+3}\right. \\
& \left.+\left(a_{i+3,6} p^{7}-a_{i+3,7} p^{6}\right) q^{i, i+1}\right) \wedge \tau,
\end{aligned}
$$

and

$$
\begin{aligned}
d^{\star} \varphi_{A(\mathcal{P})} & =-4 \star \varphi_{A(\mathcal{P})} \wedge \tau+\sum_{i \in \mathbb{Z}_{7}}\left(\left(a_{i, 6} p^{7}-a_{i, 7} p^{6}\right) q^{i+2, i+3, i+4}-\left(a_{i+2,6} p^{7}-a_{i+2,7} p^{6}\right) q^{i, i+3, i+4}\right. \\
& \left.+\left(a_{i+3,6} p^{7}-a_{i+3,7} p^{6}\right) q^{i, i+2, i+4}-\left(a_{i+4,6} p^{7}-a_{i+4,7} p^{6}\right) q^{i, i+2, i+3}\right) \wedge \tau .
\end{aligned}
$$


Let $\alpha_{i, j} \stackrel{\text { def }}{=} a_{i, 6} a_{j, 7}-a_{i, 7} a_{j, 6}$.

A computation gives:

$$
\begin{aligned}
\star d \varphi_{A(\mathcal{P})}= & \sum_{i \in \mathbb{Z}_{7}}\left[\left(-3 u_{i+2}-u_{i}\left(-\alpha_{i, i+2}+\alpha_{i+4, i+3}+\alpha_{i+5, i+1}\right)+u_{i+3}\left(-\alpha_{i+6, i+1}+\alpha_{i+3, i+2}+\alpha_{i+4, i}\right)\right.\right. \\
& \left.+u_{i+1}\left(\alpha_{i+6, i+3}+\alpha_{i+1, i+2}+\alpha_{i+5, i}\right)\right) q^{i+4, i+5, i+6} \\
& +\left(3 u_{i+4}-u_{i}\left(\alpha_{i, i+4}+\alpha_{i+2, i+3}+\alpha_{i+6, i+1}\right)+u_{i+1}\left(-\alpha_{i+1, i+4}-\alpha_{i+5, i+3}+\alpha_{i+6, i}\right)\right. \\
& \left.-u_{i+3}\left(-\alpha_{i+5, i+1}-\alpha_{i+2, i}+\alpha_{i+3, i+4}\right)\right) q^{i+2, i+5, i+6} \\
& +\left(-3 u_{i+5}+u_{i+3}\left(\alpha_{i+4, i+1}+\alpha_{i+6, i}+\alpha_{i+3, i+5}\right)+u_{i}\left(\alpha_{i, i+5}+\alpha_{i+2, i+1}-\alpha_{i+6, i+3}\right)\right. \\
& \left.-u_{i+1}\left(\alpha_{i+2, i}+\alpha_{i+4, i+3}-\alpha_{i+1, i+5}\right)\right) q^{i+2, i+4, i+6} \\
& +\left(3 u_{i+6}-u_{i+3}\left(-\alpha_{i+5, i}+\alpha_{i+2, i+1}+\alpha_{i+3, i+6}\right)-u_{i}\left(\alpha_{i+5, i+3}+\alpha_{i, i+6}-\alpha_{i+4, i+1}\right)\right. \\
& \left.+u_{i+1}\left(-\alpha_{i+4, i}-\alpha_{i+1, i+6}+\alpha_{i+2, i+3}\right)\right) q^{i+2, i+4, i+5} \\
& +\left(u_{i+6}\left(-\alpha_{i, i+2}+\alpha_{i+4, i+3}+\alpha_{i+5, i+1}\right)-u_{i+3}\left(\alpha_{i+5, i+2}+\alpha_{i, i+1}+\alpha_{i+4, i+6}\right)\right. \\
& \left.\left.+u_{i+2}\left(\alpha_{i+5, i+3}+\alpha_{i, i+6}-\alpha_{i+4, i+1}\right)-u_{i+1}\left(-\alpha_{i, i+3}-\alpha_{i+4, i+2}+\alpha_{i+5, i+6}\right)\right) q^{i, i+4, i+5}\right] .
\end{aligned}
$$

Now one can use Lemma 3.4 to check which classes $\varphi_{A(\mathcal{P})}$ belongs to. As for the class $\mathcal{W}_{2} \oplus \mathcal{W}_{3} \oplus \mathcal{W}_{4}$, one obtains

$$
0=d \varphi_{A(\mathcal{P})} \wedge \varphi_{A(\mathcal{P})}=\sigma \wedge \tau
$$

where $\sigma$ is a 6-form on $S^{6} \times S^{1}$ with constant coefficients with respect to $\mathcal{P}$, and this is easily seen to be impossible. The existence of a 1-form $\beta$ on $S^{6} \times S^{1}$ such that $d^{\star} \varphi_{A(\mathcal{P})}=\beta \wedge \varphi_{A(\mathcal{P})}$ implies that

$$
\alpha_{i, i+1}+\alpha_{i+5, i+2}-\alpha_{i+6, i+4}=0 \quad i \in \mathbb{Z}_{7} .
$$

This system has no solution, so that $\varphi_{A(\mathcal{P})}$ does not belong to the class $\mathcal{W}_{1} \oplus \mathcal{W}_{3} \oplus \mathcal{W}_{4}$. The above system comes out also requiring the existence of a 1-form $\alpha$ and a function $f$ on $S^{6} \times S^{1}$ such that $d \varphi_{A(\mathcal{P})}=\alpha \wedge \varphi_{A(\mathcal{P})}+f^{\star} \varphi_{A(\mathcal{P})}$, hence $\varphi_{A(\mathcal{P})}$ does not belong to $\mathcal{W}_{1} \oplus \mathcal{W}_{2} \oplus \mathcal{W}_{4}$. Finally, ${ }^{\star} d \varphi_{A(\mathcal{P})} \wedge \varphi_{A(\mathcal{P})} \neq 0$ by a direct computation.

If $\operatorname{det} A=-1$, some signs in formulas are reversed, but the same impossible conditions are obtained.

\section{Appendix: structure equations for $\mathcal{P}$ on $S^{m} \times S^{n}$}

As anticipated in Remark 2.2, we eventually describe the structure equations for the parallelization $\mathcal{P}$ on $S^{m} \times S^{n}$ when $n \geq 3$. We already used them in Theorem 5.1.

Using the following abbreviations one gets:

$$
\begin{aligned}
& X_{m} \stackrel{\text { def }}{=} \sum_{j=1}^{n+1} y_{j} p_{m-1+j}, \\
& X_{m+1} \stackrel{\text { def }}{=} \sum_{j=1}^{n+1} t_{j} p_{m-1+j}, \\
& C_{j, k} \stackrel{\text { def }}{=} y_{j} t_{k}-y_{k} t_{j} \quad j, k=1, \ldots, n+1, \\
& D_{j, k} \stackrel{\text { def }}{=} 2 C_{j, k} \underbrace{\mp \delta_{k, j \pm 1}}_{j \text { odd }} \underbrace{ \pm \delta_{j, k \pm 1}}_{k \frac{\text { odd }}{\text { even }}} \quad j, k=1, \ldots, n+1 .
\end{aligned}
$$


One gets also:

$$
\begin{aligned}
{\left[p_{i}, p_{j}\right] } & =x_{i} p_{j}-x_{j} p_{i} \quad i, j=1, \ldots, m-1, \\
{\left[p_{i}, p_{m-1+j}\right] } & =-\left(y_{j} x_{m}+t_{j} x_{m+1}\right) p_{i} \underbrace{\mp x_{i} p_{m-1+j \pm 1}}_{j \text { odd }}+x_{i} y_{j} X_{m}+x_{i} t_{j} X_{m+1} \quad i=1, \ldots, m-1, j=1, \ldots, n+1, \\
{\left[p_{m-1+j}, p_{m-1+k}\right] } & =D_{j, k} \sum_{i=1}^{m-1} x_{i} p_{i}+y_{j} p_{m-1+k}-y_{k} p_{m-1+j}+\left(x_{m} D_{j, k}-x_{m+1} C_{j, k}\right) X_{m}+\left(\left(x_{m+1}-1\right) D_{j, k}+x_{m} C_{j, k}\right) X_{m+1} \\
& +\underbrace{\left(\mp y_{j} x_{m} \mp t_{j} x_{m+1} \pm t_{j}\right) p_{m-1+k \pm 1}}_{k \frac{\text { odd }}{\text { even }}}+\underbrace{\left( \pm y_{k} x_{m} \pm t_{k} x_{m+1} \mp t_{k}\right) p_{m-1+j \pm 1}}_{j \text { odd }} \quad j, k=1, \ldots, n+1 .
\end{aligned}
$$

Acknowledgement: The authors were supported by the group GNSAGA of INdAM and by the PRIN Project of MIUR "Varietà reali e complesse: geometria, topologia e analisi armonica". M. P. was also supported by Università di Chieti-Pescara, Dipartimento di Economia. P. P. was also supported by Sapienza Università di Roma Project "Polynomial identities and combinatorial methods in algebraic and geometric structures".

\section{References}

[1] I. Agricola. The Srní lectures on non-integrable geometries with torsion. Arch. Math. (Brno), 42(suppl.):5-84, 2006.

[2] D.V. Alekseevskij. Riemannian spaces with exceptional holonomy groups. Funkts. Anal. Prilozh., 2(2):1-10, 1968.

[3] J. C. Baez. The octonions. Bull. Amer. Math. Soc. (N.S.), 39(2):145-205, 2002.

[4] L. Bagaglini, M. Fernández, and A. Fino. Coclosed $\mathrm{G}_{2}$-structures inducing nilsolitons. Forum Math., 30(1):109-128, 2018.

[5] R. B. Brown and A. Gray. Riemannian Manifolds with Holonomy Group Spin(9). In Differential Geometry, in honor of K. Yano, pages 41-59, Kinokuniya, Tokyo, 1972.

[6] M. Bruni. Sulla parallelizzazione esplicita dei prodotti di sfere. Rend. di Mat., serie VII, 12:405-423, 1992.

[7] F. M. Cabrera. On Riemannian manifolds with Spin(7)-structure. Publ. Math. Debrecen, 46:271-283, 1995.

[8] F. M. Cabrera. On Riemannian manifolds with $G_{2}$-structure. Bollettino U.M.I., sezione A, 7:99-112, 1996.

[9] F. M. Cabrera. Orientable Hypersurfaces of Riemannian Manifolds with Spin(7)-Structure. Acta Math. Hung., 76(3):235-247, 1997.

[10] E. Calabi and B. Eckmann. A class of compact, complex manifolds wich are not algebraic. Ann. of Math., 58:494-500, 1953.

[11] D. Conti and M. Fernández. Nilmanifolds with a calibrated $G_{2}$-structure. Differential Geom. Appl., 29(4):493-506, 2011.

[12] M. Castrillón López, P. M. Gadea, and I. V. Mykytyuk. The canonical eight-form on manifolds with holonomy group Spin(9). Int. J. Geom. Methods Mod. Phys., 7(7):1159-1183, 2010.

[13] M. Castrillón López, P. M. Gadea, and I. V. Mykytyuk. On the explicit expressions of the canonical 8-form on Riemannian manifolds with Spin(9) holonomy. Abh. Math. Semin. Univ. Hambg., 87(1):17-22, 2017.

[14] M. Fernández. A Classification of Riemannian Manifolds with Structure Group Spin(7). Ann. di Mat. Pura e Appl., 148:101$122,1986$.

[15] M. Fernández. An example of a compact calibrated manifold associated with the exceptional Lie group $G_{2}$. J. Differential Geom., 26(2):367-370, 1987.

[16] M. Fernández. A family of compact solvable $G_{2}$-calibrated manifolds. Tohoku Math. J. (2), 39(2):287-289, 1987.

[17] M. Fernández and A. Gray. Riemannian manifolds with structure group G2. Ann. Mat. Pura Appl., IV. Ser., 132:19-45, 1982.

[18] A. Fino and A. Raffero. Closed $G_{2}$-structures on non-solvable Lie groups. 2017. arXiv:1712.09664 [math.DG].

[19] M. Freibert. Calibrated and parallel structures on almost Abelian Lie algebras. 2013. arXiv:1307.2542 [math.DG].

[20] T. Friedrich. Weak Spin(9)-structures on 16-dimensional Riemannian manifolds. Asian J. Math., 5(1):129-160, 2001.

[21] T. Friedrich. On types of non-integrable geometries. In The proceedings of the 22nd winter school "Geometry and physics", Srní, Czech Republic, January 12-19, 2002, pages 99-113. Palermo: Circolo Matemàtico di Palermo, 2003.

[22] T. Friedrich. Spin(9)-structures and connections with totally skew-symmetric torsion. J. Geom. Phys., 47(2-3):197-206, 2003.

[23] A. Gray and L. M. Hervella. The Sixteen Classes of Almost Hermitian Manifolds and Their Linear Invariants. Ann. Mat. Pura Appl., IV. Ser., 123:35-58, 1980.

[24] R. Harvey and H. B. Lawson. Calibrated geometries. Acta Math., 148:47-157, 1982.

[25] S. Ivanov, M. Parton, and P. Piccinni. Locally conformal parallel $G_{2}$ and $\operatorname{Spin}(7)$ manifolds. Math. Res. Lett., 13(2-3):167-177, 2006. 
[26] D. D. Joyce. Compact manifolds with special holonomy. Oxford Mathematical Monographs. Oxford: Oxford University Press. xii, 436 p., 2000.

[27] M. Kervaire. Courbure intégrale généralisée et homotopie. Math. Ann., 131:219-252, 1956.

[28] J. Kotrbatý. Octonion-valued forms and the canonical 8-form on Riemannian manifolds with a Spin(9)-structure. 2018. arXiv:1808.02452 [math.RT].

[29] L. Ornea, M. Parton, P. Piccinni, and V. Vuletescu. Spin(9) geometry of the octonionic Hopf fibration. Transformation Groups, 18(3):845-864, 2013.

[30] M. Parton. Hermitian and special structures on products of spheres. PhD thesis, Dipartimento di Matematica, Università degli Studi di Pisa, 2001. http://www.sci.unich.it/ parton/rice/main.pdf.

[31] M. Parton. Old and new structures on products of spheres. In Global differential geometry: the mathematical legacy of Alfred Gray (Bilbao, 2000), pages 406-410. Amer. Math. Soc., Providence, RI, 2001.

[32] M. Parton. Explicit parallelizations on products of spheres and Calabi-Eckmann structures. Rend. Ist. Mat. Univ. Trieste, 35(1-2):61-67, 2003.

[33] P. Piccinni. On the cohomology of some exceptional symmetric spaces. In Special metrics and group actions in geometry, volume 23 of Springer INdAM Ser., pages 291-305. Springer, Cham, 2017.

[34] P. Piccinni. On some Grassmannians carrying an even Clifford structure. Differential Geom. Appl., 59:122-137, 2018.

[35] M. Parton and P. Piccinni. Spin(9) and almost complex structures on 16-dimensional manifolds. Ann. Global Anal. Geom., 41(3):321-345, 2012.

[36] M. Parton and P. Piccinni. Spheres with more than 7 vector fields: All the fault of Spin(9). Linear Algebra Appl., 438(3):11131131, 2013.

[37] M. Parton and P. Piccinni. The even Clifford structure of the fourth Severi variety. Complex Manifolds, 2(1):89-104, 2015.

[38] M. Parton and P. Piccinni. The role of spin(9) in octonionic geometry. Axioms, 7(4):72, Oct 2018.

[39] M. Parton, P. Piccinni, and V. Vuletescu. Clifford systems in octonionic geometry. Rend. Semin. Mat. Univ. Politec. Torino, 74(1-2):269-290, 2016.

[40] S. Salamon. Riemannian geometry and holonomy groups. Longman Scientific \& Technical, Essex CM20 2JE, England, 1989.

[41] E. B. Staples. A short and elementary proof that a product of spheres is parallelizable if one of them is odd. Proc. Am. Math. Soc., 18:570-571, 1967. 